\title{
Long-term prognostic value of mitral regurgitation in acute coronary syndromes
}

\author{
Anita Persson, ${ }^{1}$ Marianne Hartford, ${ }^{2}$ Johan Herlitz, ${ }^{2}$ Thomas Karlsson, ${ }^{2}$ \\ Torbjørn Omland, ${ }^{3,4}$ Kenneth Caidahl ${ }^{1,5}$
}

${ }^{1}$ Department of Clinical Physiology, Sahlgrenska Academy and University Hospital, Gothenburg, Sweden ${ }^{2}$ Department of Cardiology, Sahlgrenska Academy and University Hospital, Gothenburg, Sweden

${ }^{3}$ Department of Medicine, Akershus University Hospital, Lørenskog, Norway

${ }^{4}$ University of Oslo, Oslo, Norway

${ }^{5}$ Department of Clinical

Physiology, Karolinska University Hospital and Karolinska Institutet, Stockholm, Sweden

\section{Correspondence to} Professor Kenneth Caidahl, Department of Clinical Physiology and Molecular Medicine and Surgery, Karolinska Institutet, Karolinska University Hospital, SE-171 76 Stockholm, Sweden; kenneth.caidahl@ki.se

Accepted 10 August 2010 Published Online First 28 September 2010
ABSTRACT

Objectives To determine the additional prognostic value of mitral regurgitation (MR) over B-type natriuretic peptide (BNP), left ventricular ejection fraction (LVEF) and clinical characteristics in patients with acute coronary syndromes (ACS).

Design Long-term follow-up in a prospective ACS cohort with Doppler-assessed MR, echocardiographicallydetermined LVEF and plasma BNP levels by ELISA.

Setting Single-centre university hospital.

Patients 725 patients with ACS.

Main outcome measures Death and readmission for congestive heart failure.

Results During a median follow-up of 98 months, 235 patients $(32 \%)$ died. Significant MR (grade $>1$ of 4) was found in 90 patients $(12 \%)$. In a multivariate model including MR grade $>1$, LVEF $<0.40$ and BNP $>373 \mathrm{pg}$ / $\mathrm{ml}$ (75th percentile), MR was significantly associated with long-term mortality (HR 2.28, 95\% Cl 1.67 to 3.12; $\mathrm{p}<0.0001$ ). When also adjusting for conventional risk factors, MR remained significantly associated with mortality (HR 1.53, 95\% Cl 1.06 to 2.19; $p=0.02$ ), as well as with congestive heart failure (HR $2.08,95 \% \mathrm{Cl}$ 1.29 to $3.35 ; p=0.003$ ).

Conclusions MR is common in patients with ACS, provides independent risk information and should be taken into account in the evaluation of the long-term prognosis.

Left ventricular (LV) systolic function is a major prognostic determinant in coronary artery disease, and a close relation between LV ejection fraction (LVEF) and mortality has been demonstrated. ${ }^{1-4}$ In the clinical setting, echocardiography is a commonly used method for determination of LV function. In addition to evaluation of LVEF, Doppler echocardiography also permits assessment of the degree of mitral regurgitation (MR). Although long recognised as a prognostic factor in heart failure and myocardial infarction (MI), ${ }^{5-8}$ limited data are available on the prognostic value of $M R$ in the full spectrum of acute coronary syndromes (ACS). ${ }^{9}$

Circulating levels of B-type natriuretic peptide (BNP), a cardiac-derived biomarker, are associated with LV function, and BNP has emerged as an important diagnostic tool in congestive heart failure (CHF). BNP and the N-terminal fragment (NT-proBNP) of its prohormone (proBNP) have also been shown to be independently predictive of mortality in patients with $\mathrm{ACS}^{3}{ }^{4}$ as well as in patients with stable coronary disease. ${ }^{2} 10$ Notably, in patients with asymptomatic or symptomatic
MR and decreased LVEF, BNP has been shown to increase in proportion to the severity of $M R^{11}$ Moreover, in a study of consecutive patients with CHF, a significant association between higher levels of BNP and moderate to severe MR was observed, ${ }^{12}$ suggesting that some of the prognostic merit of BNP may be ascribed to its reflection of the magnitude of MR. It is unknown, however, whether indices of LV systolic function, MR severity and BNP levels provide independent and incremental prognostic information to each other. Thus, in the present study we first sought to evaluate whether MR adds prognostic information above that obtained from BNP and LVEF in ACS. Second, we assessed whether the presence of significant $M R$, increased BNP levels and decreased LVEF, alone or in combination, provided prognostic information above and beyond conventional clinical risk factors for the long-term outcome in ACS.

\section{METHODS}

\section{Patient population}

Consecutive patients admitted to the coronary care unit of the Sahlgrenska University Hospital, Gothenburg, Sweden with suspected ACS and evaluated for participation in a study of prognosis and its prediction in ACS, ${ }^{13}$ were included between December 1996 and March 2001. To enable longterm follow-up, only patients aged $<80$ years and living within the catchment area of the hospital were eligible. Patients transferred from other hospitals for tertiary care were not included. The main exclusion criteria were severe non-coronary artery disease associated with a life expectancy of $<1$ year or unwillingness to participate. A patient could only be included once.

The suspicion of ACS had to be supported by ECG changes on admission, biochemical markers above the upper reference level or previously recognised coronary artery disease. ${ }^{13}$

Based on ECG and biochemical markers of myocardial ischaemia and necrosis, the patients were diagnosed as ST-segment elevation MI (STEMI), non-STEMI or unstable angina pectoris (UAP). STEMI was further subdivided according to anterior (including lateral), inferior (including posterior) and unspecified locations.

Information on earlier hospital admissions, risk factors and medication was obtained from hospital medical records and personal interviews. Date of death and survival confirmation at 1 January 2008 was obtained from the Swedish National Population Registry, and information on rehospitalisation for CHF (International Statistical Classification of online under the BMJ Journals unlocked scheme, see http:// heart.bmj.com/site/about/ unlocked.xhtml 
Disease, Ninth Revision (ICD-9) code 428 or Tenth Revision (ICD-10) code 150) up to January 2008 from the Swedish Hospital Discharge Register.

\section{Blood samples}

Peripheral blood samples for plasma BNP determination were obtained in the acute phase within $24 \mathrm{~h}$ of hospital admission by direct venipuncture of an antecubital vein after the patient had been in the supine position for $>30 \mathrm{~min}$. The samples were drawn into prechilled ethylenediamine tetraacetic acid (EDTA) tubes, immediately placed on ice and centrifuged at $3000 \mathrm{rpm}$ within $1 \mathrm{~h}$. EDTA plasma samples were aspirated and stored at $-80^{\circ} \mathrm{C}$ until analysis (median (25th, 75th percentiles) $58(46,81)$ months). BNP levels in plasma were measured by a fluorescence immunoassay from Biosite Diagnostics (San Diego, California, USA). Interassay and intra-assay coefficients of variation for the BNP measurements were $9-12 \%$ in the low, medium and high ranges. There was a weak inverse relation between BNP levels and days of storage $(\mathrm{r}=-0.076, \mathrm{p}=0.042)$. Glomerular filtration rate was estimated by the Cockroft and Gault formula. Renal dysfunction was defined as calculated creatinine clearance $<60 \mathrm{ml} / \mathrm{min}$.

\section{Echocardiography}

Echocardiographic investigations including continuous wave Doppler recordings were performed by an experienced operator within 5 days after hospital admissions. Biplane LVEF was calculated by the disc summation method and tracings were checked in motion mode for accuracy, as described previously. 314 LVEF $<0.40$ was regarded as reduced systolic LV function. ${ }^{15}$

The grade of MR was determined from colour and continuous wave Doppler recordings based on the regurgitation flow intensity and classified into five grades. ${ }^{16}$ Grade 0 denoted no regurgitation or regurgitation limited to a small part of early systole; grade 1 indicated mild regurgitation weakly visible during the whole of systole; grade 2 was moderate regurgitation clearly visible throughout systole; grade 3 was defined by large regurgitation intensely visible during the whole of systole; and grade 4 signified severe regurgitation as found, for example, in chordal rupture with a large intensity and altered shape of the spectral Doppler profile (sharp and often a visible $V$ wave effect rather than a rounded profile). In grades $3-4$ the regurgitation usually reached the roof of the left atrium and, particularly in grade 4 , swirled around. In grade 3-4 there was also usually a volume load on the left ventricle and reversed systolic pulmonary venous flow.

\section{Statistical analyses}

Categorical variables are reported as percentages and continuous variables as medians. We used dichotomised BNP ( $>75$ th percentile), LVEF $(<0.40)$ and MR (grade $>1$ ) for the analyses. A score defined as the number of these three factors present was also calculated and analysed.

The association between dichotomised BNP, LVEF and MR and baseline demographic characteristics and clinical risk factors was assessed by the Fisher exact test for proportions and the Mann-Whitney U test for continuous/ordered variables.

The Kaplan-Meier method was used for estimation of cumulative mortality curves. In the figures these are only shown for the first 10 years, although the complete follow-up information until 1 January 2008 was used to calculate HRs and p values. Univariate mortality comparisons were performed using the log rank test, and multivariate mortality analyses as well as HR calculations (with corresponding 95\% CIs) were performed using the Cox proportional hazards model. In the multivariable analyses we adjusted for potential confounders deemed to be clinically relevant (age, gender, smoking, diabetes, hypertension, hypercholesterolaemia, prior MI, prior angina, prior CHF, prior percutaneous coronary intervention/coronary artery bypass grafting, estimated creatinine clearance and diagnosis). All $\mathrm{p}$ values are two-tailed and considered significant if $<0.05$.

\section{RESULTS}

The study population comprised 725 patients (515 men and 210 women) of mean \pm SD age $65 \pm 10$ years with STEMI $(n=343)$,

Table 1 Baseline characteristics of patients above or below the respective cut-off levels for MR grade, LVEF and plasma BNP

\begin{tabular}{|c|c|c|c|c|c|c|c|}
\hline & $\begin{array}{l}\text { All patients } \\
(\mathrm{n}=725)\end{array}$ & $\begin{array}{l}M R>1 / \leq 1 \\
(n=90 / 635)\end{array}$ & p Value & $\begin{array}{l}\text { LVEF }<0.40 / \geq 0.40 \\
(n=110 / 615)\end{array}$ & p Value & $\begin{array}{l}\text { BNP >373/ } \leq 373 \\
(n=180 / 545)\end{array}$ & p Value \\
\hline Mean age (years) & 65 & $70 / 64$ & $<0.0001$ & $70 / 64$ & $<0.0001$ & $70 / 63$ & $<0.0001$ \\
\hline Female (\%) & 29 & $39 / 28$ & 0.05 & $24 / 30$ & 0.17 & $37 / 27$ & 0.01 \\
\hline $\mathrm{Ml}$ & 20 & $36 / 18$ & 0.0002 & $36 / 17$ & $<0.0001$ & $29 / 17$ & 0.001 \\
\hline Angina pectoris & 40 & $59 / 38$ & 0.0002 & $43 / 40$ & 0.60 & $47 / 38$ & 0.04 \\
\hline CHF & 8 & $27 / 5$ & $<0.0001$ & $22 / 5$ & $<0.0001$ & $17 / 5$ & $<0.0001$ \\
\hline Current smoker & 34 & $27 / 35$ & 0.18 & $38 / 33$ & 0.32 & $30 / 35$ & 0.27 \\
\hline Diabetes mellitus & 18 & $26 / 17$ & 0.04 & $18 / 18$ & 0.89 & $21 / 17$ & 0.26 \\
\hline Hypertension & 40 & $51 / 39$ & 0.03 & $42 / 40$ & 0.75 & $51 / 37$ & 0.0008 \\
\hline Hypercholesterolaemia & 28 & $31 / 28$ & 0.53 & $32 / 27$ & 0.36 & $24 / 29$ & 0.22 \\
\hline $\mathrm{GFR}<60 \mathrm{ml} / \mathrm{min}$ & 38 & $62 / 35$ & $<0.0001$ & $53 / 36$ & 0.001 & $59 / 31$ & $<0.0001$ \\
\hline \multicolumn{8}{|l|}{ Diagnosis (\%) } \\
\hline Non-STEMI & 35 & $43 / 34$ & 0.10 & $39 / 35$ & 0.39 & $46 / 32$ & 0.0006 \\
\hline Unstable angina pectoris & 17 & $13 / 18$ & 0.37 & $12 / 18$ & 0.10 & $8 / 21$ & $<0.0001$ \\
\hline 10 -year mortality (\%) & 36.3 & $70.2 / 31.5$ & $<0.0001$ & $67.5 / 30.7$ & $<0.0001$ & $56.3 / 29.4$ & $<0.0001$ \\
\hline
\end{tabular}

BNP, brain natriuretic peptide; CABG, coronary artery bypass grafting; CHF, congestive heart failure; GFR, glomerular filtration rate; LVEF, left ventricular ejection fraction; MI, myocardial infarction; MR, mitral regurgitation, PCI, percutaneous coronary intervention; STEMI, ST segment elevation myocardial infarction. 
non-STEMI $(\mathrm{n}=256)$ or UAP $(\mathrm{n}=126)$. BNP measurements were performed in the acute phase and an echocardiographic examination with estimation of LVEF and the degree of MR was carried out within 5 days of admission. Among all the patients the $M R$ grade was inversely related to LVEF $(r=-0.32$, $\mathrm{p}<0.0001)$ and directly related to BNP $(\mathrm{r}=0.41, \mathrm{p}<0.0001)$.

Ninety patients $(12 \%)$ had an MR grade $>1,110$ patients $(15 \%)$ had an LVEF $<0.40$ and 180 patients (25\%) had BNP levels above the 75 th percentile $(373 \mathrm{pg} / \mathrm{ml})$. The characteristics of all the patients are shown in table 1 with patients divided into the following subgroups: with or without significant $M R$, with or without depressed LV function and with BNP above or below the 75th percentile. Patients in the groups with significant MR, low LVEF and high BNP were older and more often had a history of MI and CHF. Hypertension and previous angina were more common in the groups with $M R$ grade $>1$ and higher BNP, while there was no difference between the LVEF subgroups. The proportion of women was higher among patients with high BNP and tended to be high in patients with MR. Previous revascularisation and diabetes were more common in patients with $M R$ than in those without. There were no differences in index diagnosis between the MR groups or between the LVEF groups, but non-STEMI was more common in patients with high BNP levels than in those with lower levels, while the opposite was true for UAP. In the subgroups of STEMI, anterior location was associated with lower LVEF $(p<0.0001)$ and a higher BNP level $(p=0.001)$ but lower MR grade $(p=0.027)$ than inferior location. As shown in table 1 , in patients with larger MR the proportion of anterior MI was lower and the proportion of inferior MI tended to be higher than in those with minor MR. On the other hand, with LVEF $<0.40$ anterior MI was more prevalent and inferior MI was less prevalent than with $L V E F \geq 0.40$.

Two hundred and thirty-five patients (32.4\%) died during a median (25th and 75th percentiles) follow-up time of 98 (75, 117) months. MR, reduced LVEF and high BNP levels were all significantly associated with long-term mortality, as shown in figure 1 and table 2 .

In a model that included MR, LVEF and BNP, all three variables remained significant predictors of mortality (table 2, multivariate model 1). After adjustment for clinical confounders, each variable also independently predicted death (multivariate model 2). Furthermore, when MR, LVEF and BNP were forced into the same model together with clinical variables (multivariate model 3), all three factors remained significantly associated with long-term mortality (HR for MR 1.53 (95\% CI 1.06 to 2.19), $p=0.02$; HR for LVEF 1.94 (95\% CI 1.38 to 2.73), $\mathrm{p}=0.0001$; and HR for BNP 1.45 (95\% CI 1.06 to 1.99), $\mathrm{p}=0.02$, table 2). The C-statistic (81 months of follow-up) for $M R$ alone was 0.594 compared with 0.669 for MR+LVEF and 0.711 for $\mathrm{MR}+\mathrm{LVEF}+\mathrm{BNP}$.

Figure 2 shows the mortality curves for patients having none, one or $2-3$ of significant MR, low LVEF and high BNP, and illustrates how the mortality increases with a higher score (figure $2 \mathrm{~A}$ ). The combination of the non-invasive variables without BNP is also shown (figure $2 \mathrm{~B}$ ).

$M R, L V E F$ and BNP all showed a significant univariate association with readmission to hospital for CHF (table 2). After adjustment for potential confounders and taken into the same model, MR (HR 2.08, 95\% CI 1.29 to 3.35, p=0.003) and LVEF (HR 3.41, 95\% CI 2.14 to $5.42, \mathrm{p}<0.0001)$ still remained significant predictors of readmission to hospital for CHF, while a trend in the same direction was seen for BNP (HR 1.51, 95\% CI 0.96 to 2.33, $\mathrm{p}=0.07$ ).
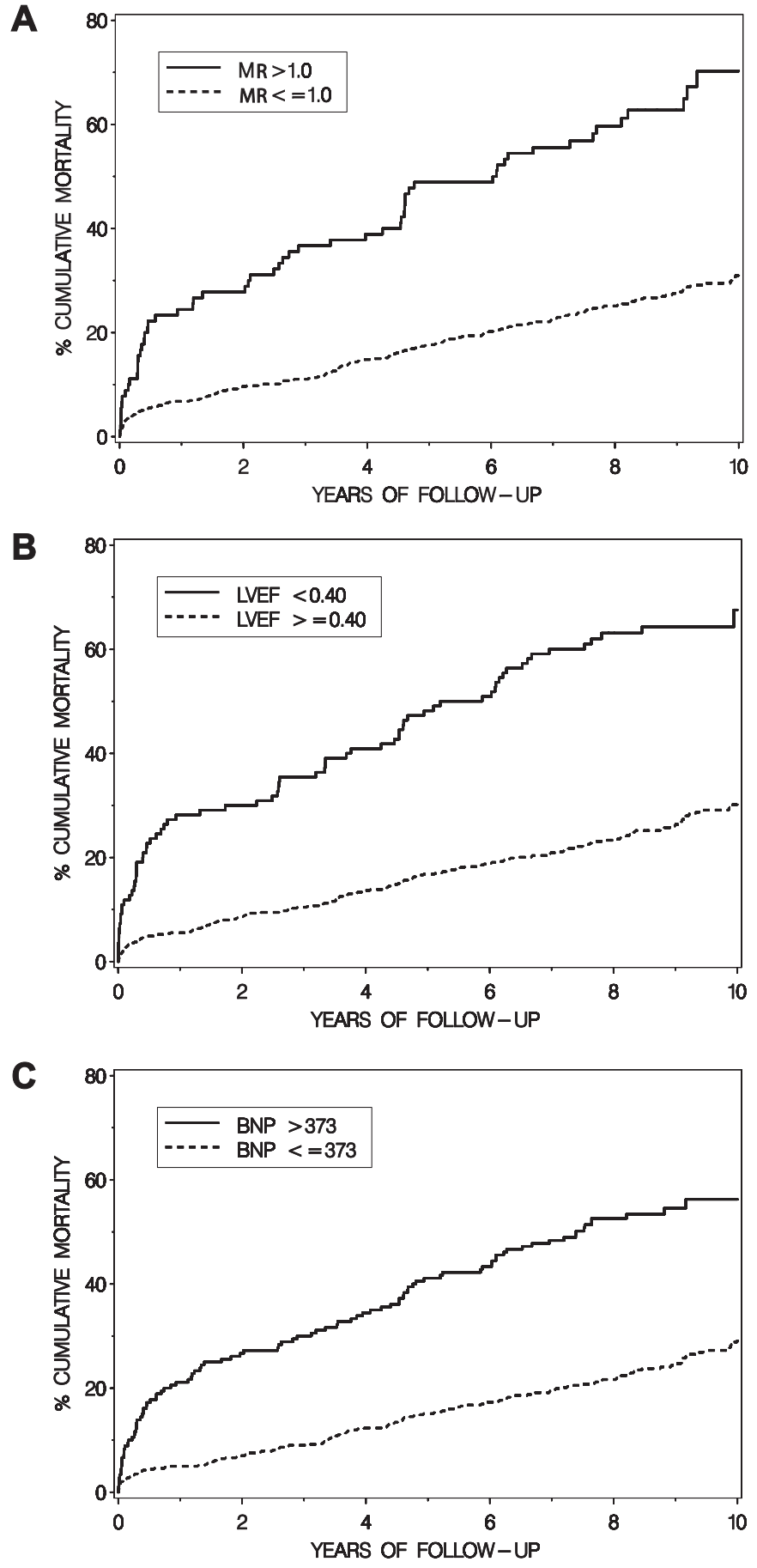

Figure 1 Kaplan-Meier curves showing the cumulative incidence of death in relation to $(A)$ mitral regurgitation $(M R)$ grade $>1$ or $\leq 1$; $(B)$ left ventricular ejection fraction (LVEF) $<0.40$ or $\geq 0.40$; and (C) B-type natriuretic peptide $(B N P)>373 \mathrm{pmol} / \mathrm{l}$ (75th percentile) or $\leq 373 \mathrm{pmol} / \mathrm{I}$ in patients with acute coronary syndromes.

\section{DISCUSSION}

In this prospective outcome study of patients with ACS, the presence of a significant MR had prognostic implications and provided independent information over that obtained from the combination of reduced LVEF, high BNP and clinical risk factors. Thus, $M R$ grade $>1$ independently predicted long-term mortality as well as readmission to hospital for CHF. 
Table 2 HRs (95\% Cls) for the association of long-term mortality to baseline MR, LVEF and BNP

\begin{tabular}{|c|c|c|c|c|c|c|c|c|}
\hline & Univariate & p Value & $\begin{array}{l}\text { Multivariate model } 1 \\
\text { including MR, LVEF } \\
\text { and BNP (or MR/LVEF* } \\
\text { and BNP) }\end{array}$ & p Value & $\begin{array}{l}\text { Multivariate model } 2 \\
\text { including MR or LVEF or } \\
\text { BNP or MR/LVEF* }+ \\
\text { confounders } \dagger\end{array}$ & p Value & $\begin{array}{l}\text { Multivariate model } 3 \\
\text { including MR, LVEF } \\
\text { and BNP (or MR/LVEF* } \\
\text { and BNP) + confounders } \dagger\end{array}$ & p Value \\
\hline $\mathrm{MR}>1.0$ & 3.25 (2.41 to 4.37$)$ & $<0.0001$ & 2.28 (1.67 to 3.12$)$ & $<0.0001$ & 1.82 (1.29 to 2.59$)$ & 0.0007 & 1.53 (1.06 to 2.19$)$ & 0.02 \\
\hline LVEF $<0.40$ & 3.48 (2.63 to 4.61$)$ & $<0.0001$ & 2.38 (1.76 to 3.23$)$ & $<0.0001$ & 2.34 (1.70 to 3.22$)$ & $<0.0001$ & 1.94 (1.38 to 2.73$)$ & 0.0001 \\
\hline $\mathrm{BNP}>373 \mathrm{pmol} / \mathrm{l}$ & 2.86 (2.20 to 3.72$)$ & $<0.0001$ & 1.96 (1.47 to 2.61$)$ & $<0.0001$ & $1.79(1.33$ to 2.40$)$ & 0.0001 & 1.45 (1.06 to 1.99$)$ & 0.02 \\
\hline $\mathrm{MR}>1.0$ & 3.84 (2.52 to 5.86$)$ & $<0.0001$ & 2.58 (1.66 to 4.01$)$ & $<0.0001$ & 2.49 (1.56 to 3.96$)$ & 0.0001 & 2.08 (1.29 to 3.35$)$ & 0.003 \\
\hline LVEF $<0.40$ & 5.14 (3.49 to 7.57$)$ & $<0.0001$ & 3.54 (2.33 to 5.38$)$ & $<0.0001$ & 4.14 (2.65 to 6.47$)$ & $<0.0001$ & 3.41 (2.14 to 5.42 ) & $<0.0001$ \\
\hline $\mathrm{BNP}>373 \mathrm{pmol} / \mathrm{l}$ & $3.30(2.27$ to 4.80$)$ & $<0.0001$ & 2.01 (1.34 to 3.03$)$ & 0.0008 & 2.12 (1.38 to 3.24$)$ & 0.0005 & 1.51 (0.96 to 2.38$)$ & 0.07 \\
\hline MR/LVEF score* & 3.66 (2.82 to 4.74$)$ & $<0.0001$ & 3.03 (2.29 to 4.02$)$ & $<0.0001$ & 2.91 (2.15 to 3.94$)$ & $<0.0001$ & 2.66 (1.94 to 3.65$)$ & $<0.0001$ \\
\hline
\end{tabular}

*Per step in 0-1-2 score, where MR grade $>1$ and LVEF $<0.40$ were assigned one score point each.

†Adjusted for age, gender, smoking, diabetes, hypertension, hypercholesterolaemia and history of diabetes, MI, angina, CHF, PCI/CABG, GFR $<60 \mathrm{ml} / \mathrm{min}$ and current diagnosis of $\mathrm{ACS}$ type. ACS, acute coronary syndromes; BNP, brain natriuretic peptide; CABG, coronary artery bypass grafting; CHF, congestive heart failure; GFR, glomerular filtration rate; LVEF, left ventricular ejection fraction; $\mathrm{Ml}$, myocardial infarction; $\mathrm{MR}$, mitral regurgitation, $\mathrm{PCl}$, percutaneous coronary intervention.

\section{Prognostic value of mitral regurgitation}

ACS may result in varying degrees of ischaemic injury and subsequent LV dysfunction. Numerous studies have shown that the degree of LV systolic dysfunction is a major - if not the most important-determinant of long-term outcome in ACS. ${ }^{17}$ Development of MR during ACS may be due to ischaemic injury
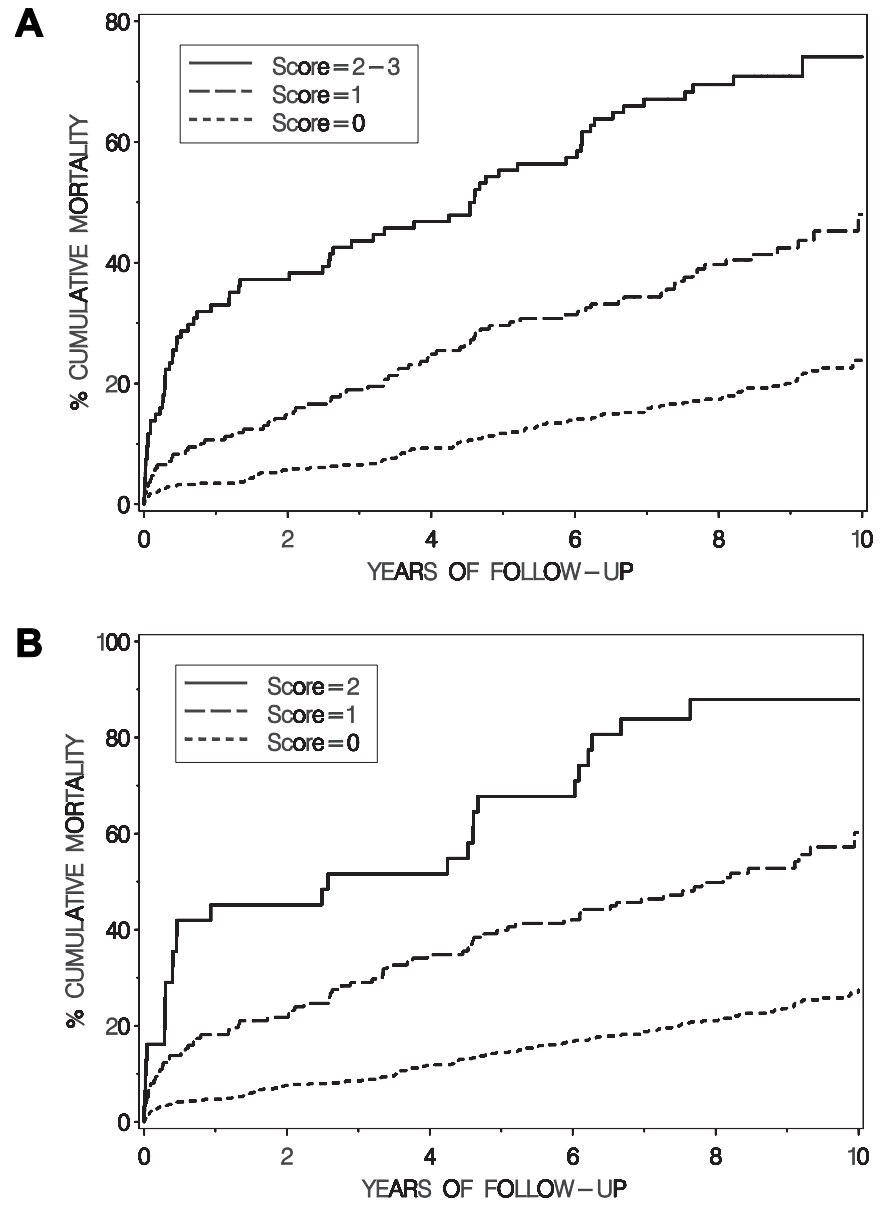

Figure 2 Cumulative incidence of death in relation to $(A)$ risk score 0,1 and 2-3 where mitral regurgitation (MR) grade $>1$, left ventricular ejection fraction (LVEF) $<0.40$ and BNP $>373 \mathrm{pmol} / \mathrm{l}$ (75th percentile) yielded one score point each; and $(B)$ risk score 0,1 and 2 where MR grade $>1$ and LVEF $<0.40$ contributed one score point each. of the papillary muscle apparatus and/or dilation of the left ventricle and has been linked to death and development of heart failure in patients with acute MI independently of LVEF and clinical confounders. ${ }^{15} 18{ }^{19}$ In addition, MR has been linked with adverse outcome in patients with STEMI undergoing a percutaneous coronary intervention. ${ }^{20}$ Our results confirm and extend the findings of these previous studies $9215 \quad 192122$ by showing that MR adds prognostic information to that of LVEF and BNP across the spectrum of ACS.

\section{BNP and mitral regurgitation}

The B-type natriuretic peptides BNP and NT-proBNP are associated with the degree of LV dysfunction following ACS, but still provide prognostic information independently of LVEF$^{3} 423$ and the Thrombolysis In MI (TIMI) risk score. ${ }^{24}$ In the present study we used BNP, which has been found to be as accurate as NT-proBNP for identifying $\mathrm{CHF}^{25}$ The volume overload and stretch of the atrial and ventricular myocardium associated with $\mathrm{MR}$ are potent stimuli for natriuretic peptide release, suggesting that in some patients the prognostic information obtained from $\mathrm{BNP}$ is partly derived from its reflection of MR grade. However, although $\mathrm{MR}$ is more common in patients with LV dysfunction, the association between MR grade and LVEF is not linear as unloading of the ventricle tends to increase LVEF. ${ }^{26}$ As confirmed in our patients, despite less LV dysfunction, inferior MI has been found to be associated with a higher incidence of MR than anterior MI, possibly due to more severe geometric changes in the mitral apparatus. ${ }^{27}$ Accordingly, the interrelationship between reduced LV function, MR grade and circulating BNP levels is a complex one. Indeed, in the current study we show for the first time that the prognostic information obtained from MR grade and BNP in ACS is complementary and significant when LVEF is also considered. In the full multivariable model which included conventional clinical risk factors, MR grade was still providing prognostic information beyond that obtained from BNP and LVEF.

\section{Prognostic value of the score system}

Despite a number of studies on the prognostic value of $M R$ as well as natriuretic peptides after an MI, we found no information on the long-term prognostic value of the combined information from MR, LVEF and BNP in ACS or MI. To determine whether a combination of the three study variables (MR, LVEF and $\mathrm{BNP}$ ) provided superior prognostic information to that 
obtained from entering individual variables, we generated a simple score system. Interestingly, a full score in the twofactor model was associated with higher risk than a score of 2-3 points in the three-factor model, suggesting that, even if inclusion of BNP in the model may be helpful for identifying low-risk patients, it seems less important for identifying high-risk patients when LVEF and MR grade are available. Our two-factor score system of MR and LVEF showed a significant association with readmission to hospital for CHF in a multivariate model including both MR/LVEF and BNP. Echocardiography allows determination of several prognostic indicators after acute $\mathrm{MI} .^{28}$ The combined information of MR and LVEF seems to contain strong supplementary prognostic information. An important advantage is that they are easily obtained in the same echocardiographic investigation, which is now often routinely performed in patients with ACS. ${ }^{17}$

\section{Strengths and limitations}

Strengths of the current study include a large contemporary patient sample and long duration of follow-up. However, there are also potential important limitations.

We stored plasma at $-80^{\circ} \mathrm{C}$ to allow uniform BNP measurements on one occasion with minimal methodological variation. Although we cannot exclude some degradation of BNP over time, only a very weak relation to days of storage was noted.

The study design used does not permit distinction between pre-existing $M R$ and $M R$ developed following the ischaemic event. Clearly, MR existing before the ACS event might also have affected subsequent prognosis. As this hospital admission was the first contact with emergency care for most patients, information on pre-existing MR grade was not generally available in our study cohort. The same limitation has been discussed by Bursi $e$ t $a l^{18}$ in a cohort study with acute MI, and is a limitation common to this and all similar studies.

Other limitations include the potential effect of a change in the acute treatment strategy of ACS during the study periodthat is, a higher proportion of patients underwent early percutaneous coronary intervention in the late part of the recruitment period than in the early part. These treatment changes may have affected the incidence of $M R$ and the predictive values of the study variables to some extent. However, acute reperfusion in patients with $\mathrm{MR}$ in relation to $\mathrm{MI}$ does not seem to reduce the mortality rate to the level noted with less MR. ${ }^{8}$ Furthermore, there is no real consensus on how to grade the magnitude of MR. The assessment of MR in our study was based on a semiquantitative grading of continuous and colour Doppler images by an experienced operator, but other methods have also been described. ${ }^{6} 18$

\section{CONCLUSION}

In summary, information concerning $M R$ grade provides important prognostic information across the spectrum of ACS. Evaluation of LV function, MR grade and BNP levels each provide complementary prognostic information to conventional clinical risk factors. A scoring system based on these three variables or $M R$ and LVEF alone represents a risk-stratifying instrument of potential clinical utility. However, its validation in a new study cohort is required before it can be generally recommended.

Funding The study was supported by the Swedish Research Council (14231), the Swedish Heart Lung Foundation, the Västra Götaland Region, the Vardal Foundation, Gothenburg University and the Gothenburg Medical Society.

\section{Competing interests None.}

Patient consent Obtained.
Ethics approval This study was conducted with the approval of the ethics committee at Gothenburg University.

Provenance and peer review Not commissioned; externally peer reviewed.

\section{REFERENCES}

1. Herlitz J, Karlson BW, Sjoland $\mathrm{H}$, et al. Long term prognosis after CABG in relation to preoperative left ventricular ejection fraction. Int J Cardiol 2000;72:163-71; discussion 73-4.

2. Kragelund C, Gronning B, Kober L, et al. N-terminal pro-B-type natriuretic peptide and long-term mortality in stable coronary heart disease. N Engl J Med 2005;352:666-75.

3. Omland T, Persson A, $\mathrm{Ng} \mathrm{L}$, et al. N-terminal pro-B-type natriuretic peptide and longterm mortality in acute coronary syndromes. Circulation 2002:106:2913-18.

4. Richards AM, Nicholls MG, Espiner EA, et al. B-type natriuretic peptides and ejection fraction for prognosis after myocardial infarction. Circulation 2003; 107:2786-92.

5. Amigoni M, Meris A, Thune JJ, et al. Mitral regurgitation in myocardial infarction complicated by heart failure, left ventricular dysfunction, or both: prognostic significance and relation to ventricular size and function. Eur Heart $J$ 2007;28:326-33.

6. Grigioni F, Detaint D, Avierinos JF, et al. Contribution of ischemic mitral regurgitation to congestive heart failure after myocardial infarction. J Am Coll Cardiol 2005; 45:260-7.

7. Lehmann KG, Francis CK, Dodge HT. Mitral regurgitation in early myocardial infarction. Incidence, clinical detection, and prognostic implications. TIMI Study Group. Ann Intern Med 1992;117:10-17.

8. Tcheng JE, Jackman JD Jr, Nelson CL, et al. Outcome of patients sustaining acute ischemic mitral regurgitation during myocardial infarction. Ann Intern Med 1992;117:18-24.

9. Perez de Isla L, Zamorano J, Quezada M, et al. Prognostic significance of functional mitral regurgitation after a first non-ST-segment elevation acute coronary syndrome. Eur Heart J 2006;27:2655-60.

10. Omland T, Richards AM, Wergeland R, et al. B-type natriuretic peptide and longterm survival in patients with stable coronary artery disease. Am J Cardiol 2005;95:24-8.

11. Sutton TM, Stewart RA, Gerber IL, et al. Plasma natriuretic peptide levels increase with symptoms and severity of mitral regurgitation. J Am Coll Cardiol 2003:41:2280-7.

12. Mayer SA, De Lemos JA, Murphy SA, et al. Comparison of B-type natriuretic peptide levels in patients with heart failure with versus without mitral regurgitation. Am J Cardiol 2004;93:1002-6.

13. Perers E, Caidahl K, Herlitz J, et al. Spectrum of acute coronary syndromes: history and clinical presentation in relation to sex and age. Cardiology 2004;102:67-76

14. Caidahl K, Kazzam E, Lidberg J, et al. New concept in echocardiography: harmonic imaging of tissue without use of contrast agent. Lancet 1998;352:1264-70

15. Lamas GA, Mitchell GF, Flaker GC, et al. Clinical significance of mitral regurgitation after acute myocardial infarction. Survival and Ventricular Enlargement Investigators. Circulation 1997:96:827-33.

16. Waagstein F, Caidahl K, Wallentin I, et al. Long-term beta-blockade in dilated cardiomyopathy. Effects of short- and long-term metoprolol treatment followed by withdrawal and readministration of metoprolol. Circulation 1989 80:551-63.

17. Lopez-Jimenez F, Goraya TY, Hellermann JP, et al. Measurement of ejection fraction after myocardial infarction in the population. Chest 2004;125:397-403.

18. Bursi F, Enriquez-Sarano M, Nkomo VT, et al. Heart failure and death after myocardial infarction in the community: the emerging role of mitral regurgitation. Circulation 2005;111:295-301.

19. Ennezat PV, Darchis J, Lamblin N, et al. Left ventricular remodeling is associated with the severity of mitral regurgitation after inaugural anterior myocardia infarction: optimal timing for echocardiographic imaging. Am Heart $J$ 2008;155:959-65.

20. Pellizzon GG, Grines CL, Cox DA, et al. Importance of mitral regurgitation inpatients undergoing percutaneous coronary intervention for acute myocardial infarction: the Controlled Abciximab and Device Investigation to Lower Late Angioplasty Complications (CADILLAC) trial. J Am Coll Cardiol 2004;43:1368-74.

21. Grigioni F, Enriquez-Sarano M, Zehr KJ, et al. Ischemic mitral regurgitation: longterm outcome and prognostic implications with quantitative Doppler assessment. Circulation 2001;103:1759-64.

22. Hillis GS, Moller JE, Pellikka PA, et al. Prognostic significance of echocardiographically defined mitral regurgitation early after acute myocardial infarction. Am Heart J 2005;150:1268-75.

23. Omland T, Aakvaag A, Bonarjee WV, et al. Plasma brain natriuretic peptide as an indicator of left ventricular systolic function and long-term survival after acute myocardial infarction. Comparison with plasma atrial natriuretic peptide and N-terminal proatrial natriuretic peptide. Circulation 1996;93:1963-9.

24. Khan So, Quinn P, Davies JE, et al. N-terminal pro-B-type natriuretic peptide is better than TIMI risk score at predicting death after acute myocardial infarction. Heart 2008;94:40-3. 
25. Gorissen C, Baumgarten R, de Groot M, et al. Analytical and clinical performance of three natriuretic peptide tests in the emergency room. Clin Chem Lab Med 2007;45:678-84.

26. Caidahl K, Larsson S, Sudow G, et al. Conservative surgery for mitral valve prolapse with regurgitation: clinical follow-up and noninvasive assessment. Eur Heart $J$ 1987:8:384-94
27. Kumanohoso T, Otsuij Y Yoshifuku $S$, et al Mechanism of higher incidence of ischemic mitral regurgitation in patients with inferior myocardial infarction: quantitative analysis of left ventricular and mitral valve geometry in 103 patients with prior myocardial infarction. J Thorac Cardiovasc Surg 2003;125:135-43.

28. Mollema SA, Nucifora G, Bax JJ. Prognostic value of echocardiography after acute myocardial infarction. Heart 2009;95:1732-45.

\section{Images in cardiology}

\section{‘Classic' supravalvular aortic stenosis}

A 16-year-old boy with a history of gradually progressive dyspnoea on exertion was referred as a case of severe aortic stenosis. On examination he was found to have a $4 / 6$ ejection systolic murmur at the base of the heart radiating to both carotids and decreased pulses on the left upper limb. EKG revealed features of left ventricular hypertrophy. Echocardiography showed normal left ventricular function with hypertrophy, tri-leaflet aortic valve with good opening of leaflets and supravalvular aortic narrowing. On diagnostic cardiac catheterisation there was no gradient across the aortic valve, but a gradient of $150 \mathrm{~mm} \mathrm{Hg}$ in the ascending aorta (Panel A). Angiography revealed tubular narrowing of the ascending aorta, dilated aortic sinuses and coronary arteries, typical of supravalvular aortic stenosis (Panel B). In addition, there was involvement of the aortic arch vessels in the form of mild ostial stenosis of the right brachiocephalic trunk with fusiform dilatation of the artery and tight ostial stenosis of the left common carotid artery. Digital subtraction angiography of the aortic arch and branch vessels confirmed these findings. Angiogram of the pulmonary arteries and the renal arteries revealed no abnormality. He lacked features suggestive of Williams-Beuren syndrome, with a normal facies and normal serum calcium levels.

This case illustrates the classic haemodynamic and angiographic features of this well-known yet uncommon entity.

\section{Salman Salahuddin, S Ramakrishnan, B Bhargava}
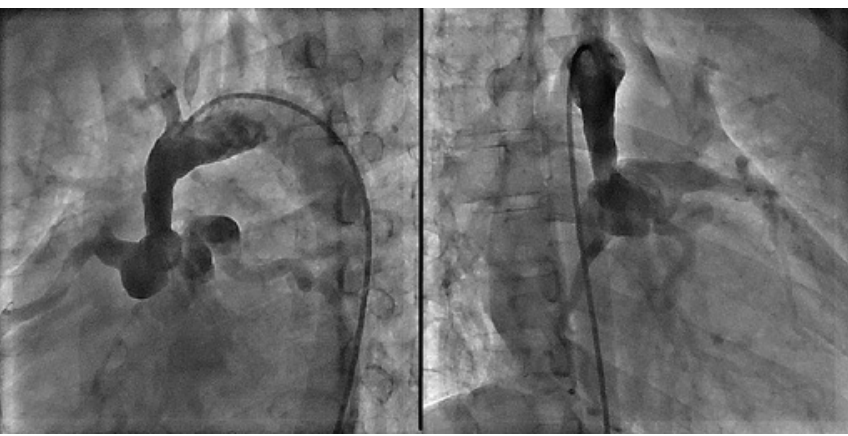

Panel B Aortic root angiogram in the left and right anterior oblique projections showing the typical tubular narrowing of the ascending aorta, dilated aortic sinuses and dilated coronary arteries. 0stial stenosis of the left common carotid artery is also visible.

Department of Cardiology, Cardiothoracic Sciences Centre, All India Institute of Medical Sciences, New Delhi, India

Correspondence to S Ramakrishnan, Department of Cardiology, 7th Floor, Cardiothoracic Sciences Centre, All India Institute of Medical Sciences,

New Delhi-110029, India; ramakgmc@rediffmail.com

- Additional data are published online only. To view these files please visit the journal online (http://heart.bmj.com)

Competing interests None.

Patient consent Obtained.

Provenance and peer review Not commissioned; not externally peer reviewed.

Published Online First 28 September 2010

Heart 2010;96:1808. doi:10.1136/hrt.2010.206235
Panel A Pullback pressure tracing from left ventricle to ascending aorta to descending aorta, showing no gradient across the aortic valve, but a gradient of $150 \mathrm{~mm} \mathrm{Hg}$ across the supravalvular ascending aorta.

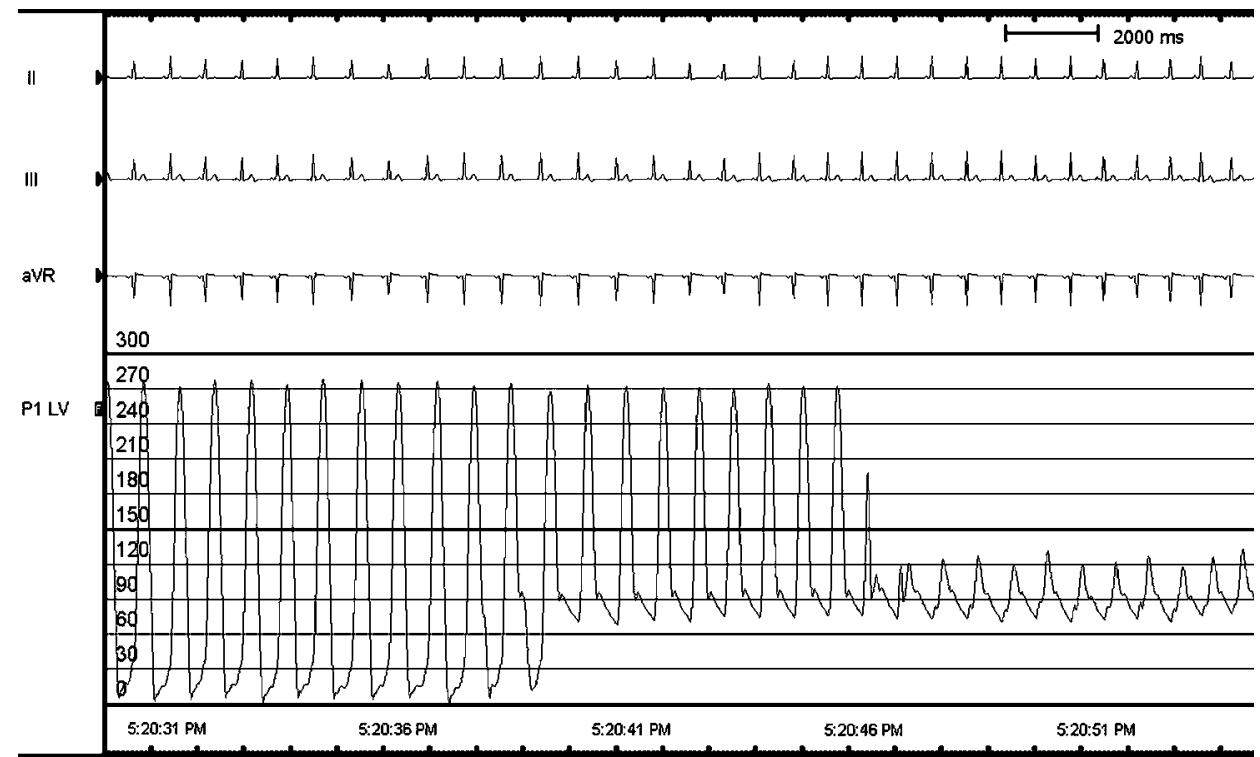

